\title{
Dryout occurrence in a helically coiled steam generator for nuclear power application
}

\author{
L. Santini ${ }^{1, \mathrm{a}}$, A. Cioncolini ${ }^{2}$, C. Lombardi $^{3}$, M. Ricotti $^{3}$ \\ ${ }^{1}$ Enel Ingegneria e Ricerca S.p.A.,Via Mantova 24, 00198 Roma, Italy \\ ${ }^{2}$ Mechanical. Aerospace and Civil Engineering, University of Manchester, Oxford Road, M13 9PL \\ Manchester, UK. \\ ${ }^{3}$ CeSNEF-Nuclear Engineering Division, Department of Energy, Deputy Head, via La Masa 34, 20156 Milano, \\ Italy
}

\begin{abstract}
Dryout phenomena have been experimentally investigated in a helically coiled steam generator tube. The experiences carried out in the present work are part of a wide experimental program devoted to the study of a GEN III+ innovative nuclear power plant [1]. The experimental facility consists in an electrically heated AISI 316L stainless steel coiled tube. The tube is 32 meters long, $12.53 \mathrm{~mm}$ of inner diameter, with a coil diameter of $1 \mathrm{~m}$ and a pitch of $0.79 \mathrm{~m}$, resulting in a total height of the steam generator of 8 meters. The thermo-hydraulics conditions for dryout investigations covered a spectrum of mass fluxes between 199 and $810 \mathrm{~kg} / \mathrm{m} 2 \mathrm{~s}$, the pressures ranges from 10.7 to $60.7 \mathrm{bar}$, heat fluxes between 43.6 to $209.3 \mathrm{~kW} / \mathrm{m} 2$.Very high first qualities dryout, between 0.72 and 0.92 , were found in the range of explored conditions, comparison of our results with literature available correlations shows the difficulty in predicting high qualities dryout in helical coils., immediately following the heading. The text should be set to 1.15 line spacing. The abstract should be centred across the page, indented $15 \mathrm{~mm}$ from the left and right page margins and justified. It should not normally exceed 200 words.
\end{abstract}

\section{Introduction}

Helically coiled once-through boilers have been widely applied in the past on several power conversion systems, ranging from supercharged fossil fuelled power stations, solar power plants, stationary nuclear reactors (AGR) and one water cooled nuclear reactor for ship power propulsion (Otto Hahn). The main advantage in such a tubular configuration is related to its compactness and capability of accommodating thermal expansions.

The hydrodynamics in a coiled tube is complicated with respect to straight tubes due to the presence of secondary flows, i.e. two counter-rotating vortices in tube cross section caused by the interaction between the centrifugal field and the variable axial velocity profile [2], [3], [4], [5].

Between the many two-phase flow phenomena involved in helical tubes, dryout occurrence is of particular importance mainly due to its reflections on

\footnotetext{
${ }^{\mathrm{a}}$ Corresponding author: lorenzo.santini@polimi.it
}

sizing and steam generator safe operation. With the name of dryout we mean the disruption of liquid film flowing in contact with tube wall associated with an annular flow regime. This phenomenon in all once through boilers is an unavoidable one and is related to a situation in which the liquid film is so thin and destabilized that breaks living the tube wall uncovered. The reduction in heat transfer coefficients that follows dryout inception is so high (especially in straight tubes) that an accurate prediction of dryout occurrence is necessary for the proper thermal sizing of the heat exchanger. Furthermore dryout location prediction is particularly important for controlling and predicting fatigue and corrosion problems, as AGR operation have shown in the past [6].

Dryout occurrence in straight vertical tubes is a point phenomenon that, on a time-averaged basis, occurs at a certain tube abscissa contemporarily in all the points around tube circumference. On the contrary dryout in coiled tubes starts at one point around tube circumference 
and then spreads over a defined length of the tube (where part of the tube is wetted and part is un-wetted) until all the circumference is dried out. A complete characterization of dryout in coils imposes, at least, the specification of three parameters [7]:

-First dryout quality and its angular position around tube circumference, i.e. the point along tube abscissa in which at least one point in the tube circumference is dried.

-Last dryout quality, i.e. the point in which all tube circumference is dried

-The degree of thermodynamic disequilibrium in the post dryout region (that extends also above $\mathrm{x}=1$ )

It is necessary to underline that the big difficulties and the high uncertainties in determining very high qualities dryout (see below) render literature results affected by a huge degree of uncertainty. Furthermore, another important aspect often neglected in open literature is dryout identification procedure, i.e. the method used by the various authors for determining dryout inception. The application of different methods for detecting dryout inception could bring to significantly different results in dryout data analysis [8].

In a vertical axis coiled tube with reasonably low pitch (as in nearly all the coiled tube steam generators), the stratifications induced by the gravity force together with the centrifugal field and the inertial force of the vapour phase play a central role in determining dryout occurrence. The complex mutual interaction between the mentioned forces determines the different roles that entrainment and redeposition play.

Regarding dryout in coils Jayanti and Berthoud [7] made a clear explanation of the various effects of externally controllable parameters, i.e. mass flux, pressure, inlet subcooling, heat flux and coil diameter on internally not directly controllable ones, i.e. entrainment and redeposition. Literature results show that mass flux effect on dryout quality in coils is not monotonic: a mass flux increase causes in some cases a dryout quality increase [9] and in other a dryout quality decreases [10]. At fixed coiled diameter mass flux mainly affects entrainment and redeposition. Entrainment is increased because of the increased shear effects between vapour phase and liquid one (this is the controlling mechanism that causes dryout quality reduction in straight tubes due to a mass flux increase), while redeposition is increased due to the increased centrifugal force that pushes the entrained droplets (that travels nearly at the same speed of the vapour core) toward the external tube wall. Depending on the dominating role of one of the two mechanisms, dryout quality in coils could increase or decrease following a mass flux increase. A more detailed discussion of mass flux effect on dryout quality could be found in Berthoud and Jayanti work [11], in which it is qualitatively shown that, if stratification effects due to gravity play a minor role, a mass flux increase causes a dryout quality increase in case of large coils diameters and high (relative) pressures. On the contrary, when gravity causes significant stratifications, i.e. with low mass fluxes and big coil diameters, the dryout inception is mainly caused by the draining and evaporation of the liquid film at the top. In this situation a mass flux increase, by affecting the liquid flow rate at the top of the tube circumference, causes also an increase in dryout quality.

The effect of wall heat flux on quality dryout is felt mainly through its impact on entrainment of liquid caused by the disruptive effect of the bubbles on liquid film created at tube wall due to nucleate boiling. In general it is possible to sentence that a heat flux increase causes a dryout quality decrease because of the increased number of bubbles pertaining to the high heat fluxes. Nevertheless this effect vanishes at low heat fluxes and very high dryout qualities (as in liquid heated helically coiled steam generators) due to the too small wall superheat characteristic of very thin films that avoid the possible formation of bubbles at tube wall. The law of the insensitivity of dryout quality to the heat flux in the region of high qualities and low heat fluxes is more valid when the pressure is low due to the larger necessary wall superheat for nucleation that characterizes low pressure flow boiling [9].

Regarding inlet subcooling there is evidence from literature that in well-established annular flow and for moderate heat fluxes, it does not influence dryout quality and, by changing two phase region length, it linearly impact on the critical heat flux.

Ünal and co-workers [12] tested three long sodium heated coiled tubes for LMFBR application. Both first and last CHF were detected and correlated with system external parameters (pressure, mass flux, inlet subcooling etc.). No attempt was made for putting in light eventual thermodynamic disequilibrium in the post dryout region. In their experimental conditions for mass fluxes higher than $850 \mathrm{~kg} / \mathrm{m} 2 \mathrm{~s}$ first dryout took place at tube intradosso (tube part nearer to coil axis), while for mass fluxes lower that $850 \mathrm{~kg} / \mathrm{m} 2 \mathrm{~s}$ it took place contemporarily at top and bottom parts of the tube. The authors do not recommend to extend the data obtained on electrically heated test sections to fluid heated ones.

Styrikovich and co-workers [10] tested one helical coiled electrically heated tube with steam-water mixture flowing inside at high pressures. The authors showed that at $x>0.2$ the critical heat flux in the coil are much higher than in a straight vertical tube and that the temperature regime in the post-dryout regime is less severe, thus indicating higher HTCs in this region. No attempt to propose a predictive correlation was done by the authors.

Jensen and Bergles [13] tested three electrically heated coiled tubes with boiling R113 inside both with and without a circumferential heat flux tilt of the tube. Both DNB and dryout type crisis were investigated and simple correlations proposed for $\mathrm{CHF}$ prediction. The authors showed that the quality region $\mathrm{CHF}$ in a coil with a flux tilt is lower than in a coil uniformly heated around the circumference.

In the present work dryout onset in a helically coiled steam generator representing one full scaled mean tube of IRIS innovative nuclear reactor steam generator has been experimentally investigated. The experimental data we think add new information regarding such an important thermohydraulic parameter in coiled once-through steam generators. A simple correlation based on the global 
approach and valid in the range of explored conditions has been proposed for first dryout quality prediction.

\section{Experimental facility}

The test section framed in the facility represents one single helical tube of IRIS SG. Coil diameter (1m) has been chosen as representative of a mean value of IRIS SG tube, while tube inner diameter $(12.53 \mathrm{~mm})$ is the commercially scheduled value nearer to IRIS real value (13.24 mm).

The facility is located at SIET (Società Italiana Esperienze Termoidrauliche) labs in Piacenza [14] and it is made by a supply section (Figure 1) and a test section (Figure 2).

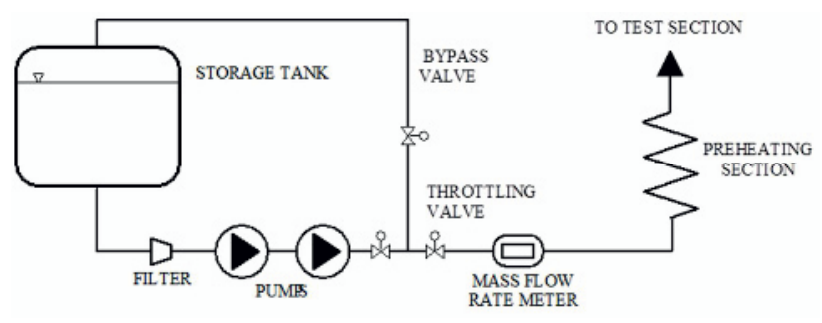

Figure 1. Scheme of the supply section

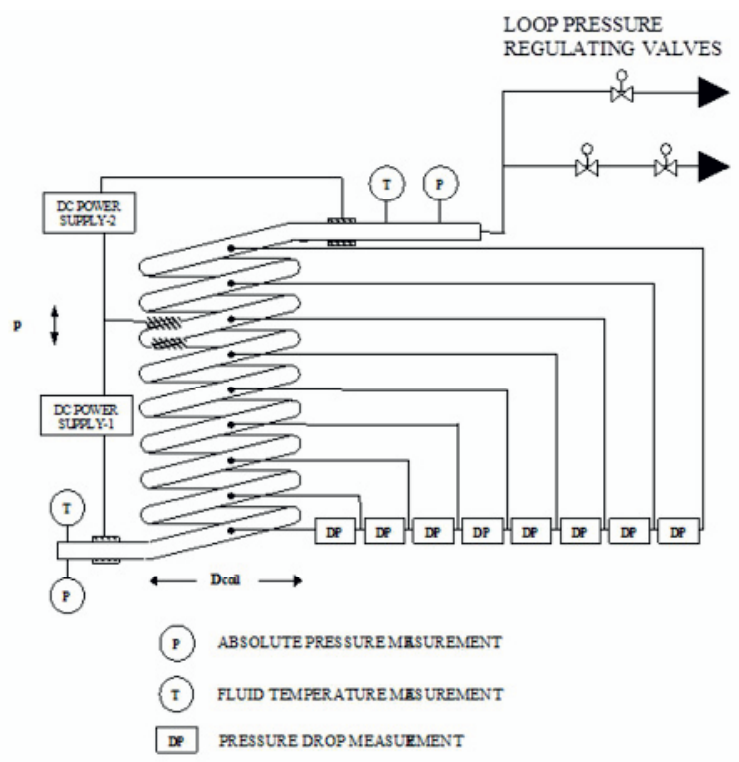

Figure 2. Scheme of the test section

The supply section feeds demineralized water from a tank to the test section, by means of a centrifugal booster pump and a feed-water pump, i.e. a volumetric threecilindrical pump with a maximum head of about 200 bar driven by an asynchronous three-phases motor. Water is demineralised via an ionic exchanger resin bed, thus obtaining an electrical conductivity value for the fluid of about $1.5 \mu \mathrm{S} / \mathrm{cm}$. The flow rate is controlled by a throttling valve positioned downwards the feed water pump and after a bypass line; the strong reduction of the supply pressure at the pump outlet avoids any two-phase flow instability in the test section. System pressure control is accomplished by acting on a throttling valve placed at the end of the steam generator, whose degree of throttle is manually imposed by acting on a pneumatic actuator until the desired pressure is reached.

An electrically heated helically coiled pre-heater is located before the test section, and allows to create the desired temperature at the inlet of the test section.

The test section in Figure 2 is electrically heated via Joule effect by DC current. Two distinct, independently controllable and contiguous sections are provided: the first one simulates the subcooling zone and the two-phase zone of the steam generator, while the second simulates the post dryout and superheating zone.

The possibility of controlling thermal flux in the two zones allowed us to roughly simulate the variation in heat flux that occurs in a real once through steam generator.

The test section consists of a stainless steel tube, curved in helical shape and connected to a lower and a upper header. The main data of the steam generator tube are listed in Table 1.

Table 1. Test section main data.

\begin{tabular}{|l|c|}
\hline Tube material & SS AISI 316 \\
\hline Inner diameter, $\mathrm{d}[\mathrm{mm}]$ & 12.53 \\
\hline Outer diameter $[\mathrm{mm}]$ & 17.24 \\
\hline Coil diameter, D [mm] & 1000 \\
\hline Coil pitch [mm] & 790 \\
\hline Tube length [m] & 32 \\
\hline Steam generator height $[\mathrm{m}]$ & 8 \\
\hline
\end{tabular}

An accurate measurement of the flow rate is obtained by a Coriolis flow-meter, placed between the pump and the pre-heater.

Bulk temperatures are measured with type " $\mathrm{K}$ " thermocouple with insulated junction. The thermocouples have $1.5 \mathrm{~mm}$ diameter and are directly immersed into the fluid, the maximum absolute uncertainty in the range of the explored temperatures is $0.7^{\circ} \mathrm{C}$. All the measurement devices have been tested and calibrated at the certified SIET labs (SIT certified).

The water pressure at heating section inlet is measured by an absolute pressure transducer; nine pressure taps are disposed nearly every four meters along the coiled tube and eight differential pressure transducers connect the pressure taps.

Wall temperatures are measured with k-class thermocouples of two groups: brazed in tube wall (Thermocoax) and manually attached on tube external wall (naked thermocouples).

A total of 128 thermocouples have been applied: 8 are brazed and disposed in 4 groups of two opposed thermocouples (INTRA and EXTRA positions in Figure $3)$; of the remaining 120 thermocouples 116 are applied in 29 groups of 4 (INTRA, EXTRA, UP and DOWN 
positions in figure 3) and 4 are applied in 2 groups of 2 (INTRA and EXTRA positions in Figure 3).

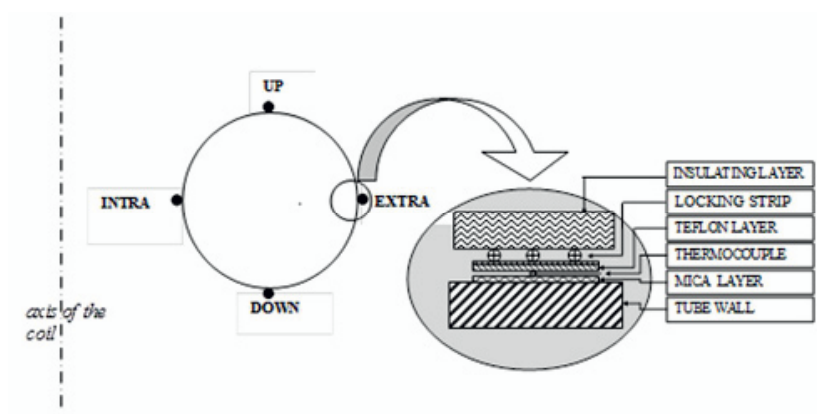

Figure 3. Manually attached Thermocouples: disposition around tube circumference and application structure

A Mica layer was positioned between tube wall and the thermocouple due to the necessity of maintaining thermocouples head electrically insulated. A Teflon layer and a locking strip are applied above the thermocouples in order to jam them.

All the measurements are acquired by a multi-channel data acquisition system with a frequency of acquisition of $4 \mathrm{~Hz}$ and stored into a computer.

The steam generator tube is carefully insulated with rock wool, and the small thermal losses were previously determined with dedicated experiences and the net power given to the fluid was determined as the difference between the electrical power and the thermal losses. This losses were measured by evaluating the flow rate and the temperature drop of hot pressurized water flowing into the steam generator and their value were correlated with the tube metal temperature.

Electric power is supplied to the steam generator via Joule effect using low voltage (a hundred Volts)-high amperage current. The electric power generator is the coupling of a AC transformer (from $130 \mathrm{kV}$ to $3 \mathrm{kV}$ ) with a Chopper that convert alternate current into (nearly) direct current.

Electrical power was obtained via separate measurement of current (by a shunt) and voltage drop along the test section by a voltmeter.

\section{Uncertainties and wall thermocouples calibration procedure}

Calibration certificates furnished by SIET labs (sit certified) give for the bulk temperatures a maximum absolute uncertainty of $0.6^{\circ} \mathrm{C}$, for the absolute pressures a maximum relative uncertainty of $0.1 \%$, for the relative pressure transducers a maximum relative uncertainty of $0.4 \%$. Coriolis flow meter has a maximum relative uncertainty on mass flow measurement of $1 \%$ in the range of explored conditions.

Wall thermocouples required a particular procedure for on site calibration using a two-phase mixture (generated in the preheater) flowing inside the test section in nearly adiabatic conditions. The goal of the calibration procedure was to quantify the thermal resistance between thermocouples hot junction and tube wall external side caused by the application procedure thanks to the known local bulk (saturation) temperature and the heat losses (previously measured with single phase runs). The problem comes from the particular procedure adopted for wall thermocouples application (see chapter on exp. Facility) that gave a different application thermal resistance for every thermocouple due to the unavoidable slight differences in locking force between the various TCs stations. The explained calibration procedure gave a contact thermal resistance for every thermocouple as a function of wall temperature. This values were used for HTCs data reduction. Wall thermocouples absolute uncertainty is estimated to be $1.5{ }^{\circ} \mathrm{C}$ for every thermocouple. This value must be combined with thermocouple application temperature drop uncertainty and with tube wall temperature jump uncertainty. The global uncertainty on HTCs, that is a function of operative conditions, has been obtained coupling all the chain of uncertainties using standard error propagation techniques and its results are reported on HTCs plots (see next).

The relative uncertainty on the net power given to the fluid, i.e. the difference between electrical power and heat losses, is a function of operative conditions, but for data reduction it has been assumed equal to the maximum calculated value, i.e. $3.5 \%$.

Test section thermodynamic equilibrium quality absolute uncertainty is a function of operative conditions (it rises with rising pressure and decreasing flow rate, due to the higher importance of thermal losses whose estimation is subject to big uncertainties), but for data reduction it has been assumed equal to the maximum calculated value, i.e. $6 \%$.

\section{Dryout identification method}

Several types of experimental procedures have been proposed in the past for thermal crisis identification. The problem of defining the thermal crisis identification method is not a secondary one due to the strong dependence of critical heat flux (CHF) value (and critical quality) on the specific adopted identification method.

It must be pointed out that in the upper range of qualities and lower range of critical heat fluxes (as in fluid heated heat exchangers), the exact location of the critical point is more and more liable to personal interpretation, thus introducing a subjective element [8].

Amongst the many definitions of critical heat flux, one can recall the following:

1-Heat flux at which the heat transfer coefficient reaches a maximum (definition suited for dryout crisis type).

2-Heat flux at which a temperature rises sufficient to burst the pressurized heated tube or to heat part of the tube to red (suited both for dryout and DNB crisis types). 3 -Heat flux at which a discontinuity occurs in the curve of the temperature of the heated wall versus heat flux.

4-Heat flux at which temperature oscillations first appear in the time-temperature recording (definition particularly suited for dryout type crisis). 
We have chosen the last mentioned method for crisis identification, being the most representative of the physical origin of dryout type crisis.

\section{Dryout investigations results}

\subsection{Introduction}

The test matrix covered a spectrum of three nominal pressures (20, 40 and 60 bar) and four mass fluxes (200, 400,600 and $800 \mathrm{~kg} / \mathrm{m} 2 \mathrm{~s}$ ), resulting in a total of 12 runs for dryout occurrence investigations. Test section inlet subcooling, i.e. the difference between saturation temperature at inlet pressure and actual inlet temperature, remained fixed at $50{ }^{\circ} \mathrm{C}$ for all the runs. Both first and last qualities dryout were investigated, no attempt were done for quantifying the degree of thermodynamic disequilibrium in the post dryout zone due to the absence of a measure of steam temperature in the post dryout region.

\subsection{Mass flux and pressure effects on dryout qualities and powers}

Figure 4 collects dryout results showing mass flux and pressure effects on first quality dryout.

A mass flux increase, in our experienced conditions, always increases first dryout quality. This is probably related to the redeposition effect of centrifugal force that dominates with respect to the entrainment effect (that increases with an increasing mass flux). The entrainment effect is the cause of dryout quality reduction experienced in straight channels caused by a mass flux increase [15].

The parameter in Figure 4 is pressure: an increasing pressure has the effect of reducing dryout quality (having fixed mass flux and inlet subcooling in term of temperature). Pressure effect is felt mainly through the properties of the fluid: at low relative pressures vapour density is lower and vapour phase is running faster. This effect increases contemporarily entrainment and redeposition and, depending on the weight of the two contributes, a pressure increase could in principle increases or decreases dryout quality in coiled tubes. Figure 4 also shows the big absolute uncertainties associated with dryout quality evaluation, as previously said, caused by the high qualities and to the uncertainties on heat losses and electrical power.

Last quality dryout dependence on mass flux and pressure is reported in Figure 5.

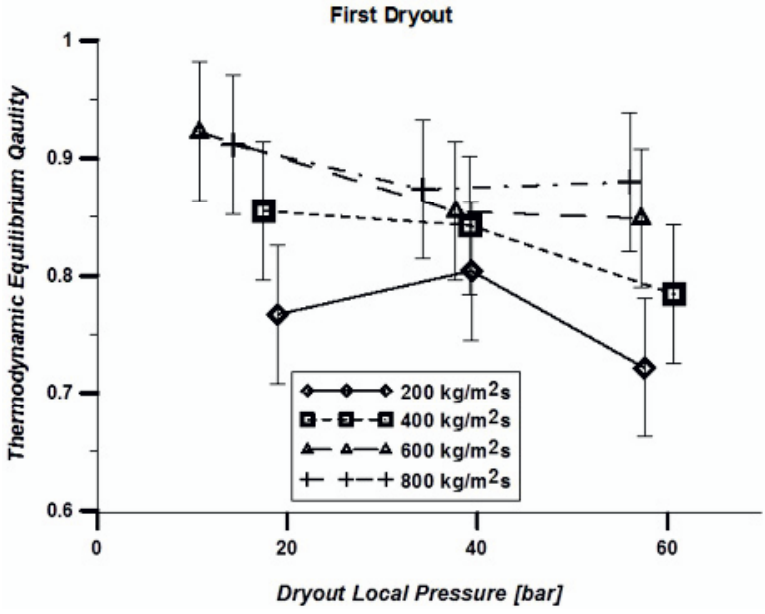

Figure 4. First quality dryout as a function of mass flux and pressure

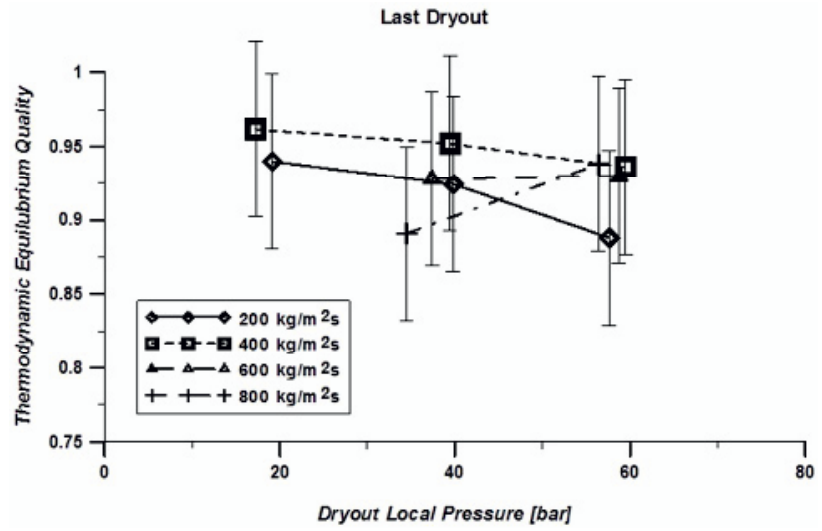

Figure 5. Last quality dryout as a function of mass flux and pressure

As it is possible to observe, the steam generator reaches the total dryout for qualities between 0.89 to 0.96 . Due to the very high uncertainties on thermodynamic quality it is difficult to infer (from our experimental results ) any law regarding last quality dryout dependence on mass flux and pressure.

Figure 6 collects mass flux effect on first dryout power. Critical power is a linearly increasing function of mass flux and is a monotonically decreasing function of system pressure.

The effect of mass flux on critical power for the last dryout is reported in Figure 7, confirming the linearity encountered for the first dryout. 


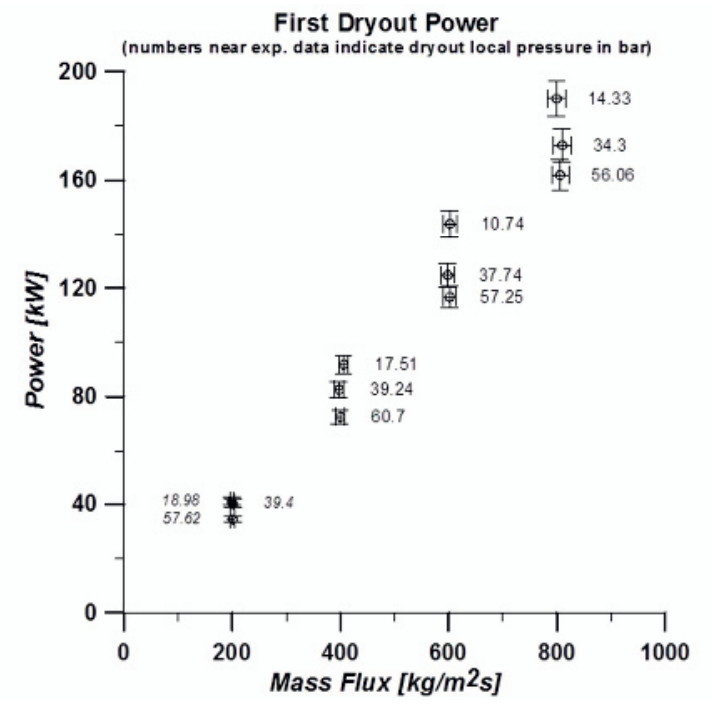

Figure 6. First dryout power as a function of mass flux and pressure

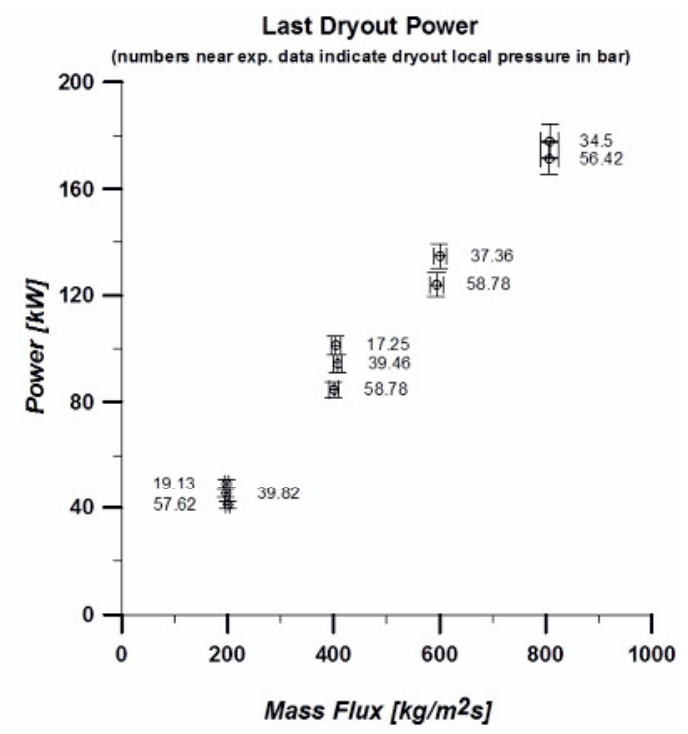

Figure 7. Last dryout power as a function of mass flux and pressure

\subsection{Dryout Quality as a function of critical power}

The choice of assuming critical power instead of critical heat flux as a controlling parameter for dryout evaluation derives from the assumption of the validity of the global hypothesis instead of the local one. The assumption of negligible local effects on dryout occurrence is reasonably valid for low heat fluxes, as in our experienced conditions. Low heat fluxes combined with high thermodynamic qualities (small film thickness), by reducing the wall superheat (with respect to saturation temperature), cause a reduction in wall nucleation effects on dryout occurrence. In fact, with high rates of bubble formation at tube wall, when the bubbles leave the liquid film they break the surface of the film and contribute to the entrainment of the liquid, resulting in a lower dryout quality for high heat fluxes (having fixed pressure and mass flux).

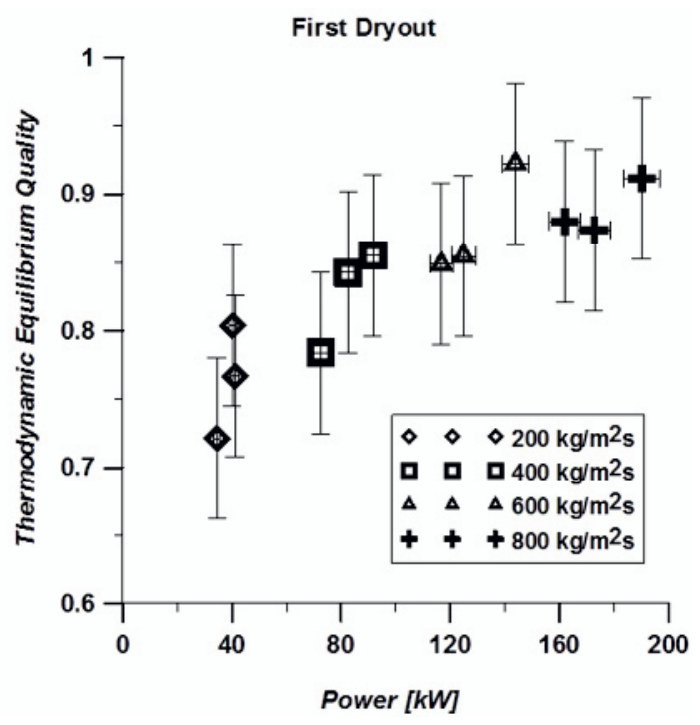

Figure 8. First dryout Quality as a function of dryout power and mass flux

On the contrary, critical power scarcely affects last dryout quality, as mass flux effect on last dryout quality investigation (Figure 6) have shown.

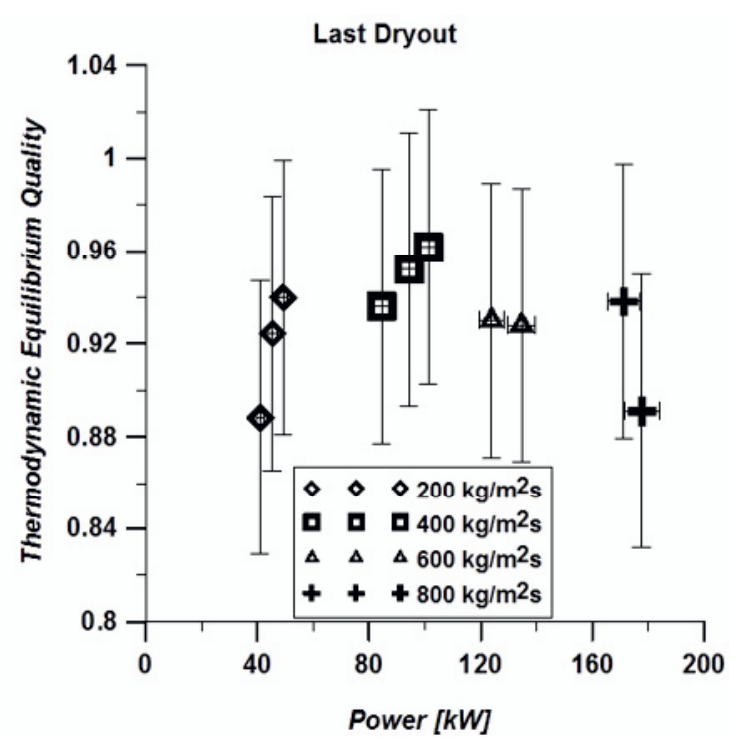

Figure 9. Last dryout Quality as a function of dryout power and mass flux

The very high thermodynamic qualities and the very low heat fluxes typical of our experiences allow us to exclude any heat flux effect on dryout occurrence. In Figs 8 and 9 the relation between first dryout quality and critical power is plotted for the experimental data obtained on our facility, both for first and for last dryout occurrence. For first quality dryout the effect of critical power is evident and we think that can be linked to the effect of mass flux on critical power: an increasing mass flux 
increases first critical power, but also first critical quality (for the assumed reasons previously explained). It results from Figure 8 that critical power effect on first dryout quality is apparent because hidden by mass flux effect on critical power.

\subsection{A correlation for first quality dryout and first critical power prediction}

Data analysis have shown that first quality dryout $(\mathrm{xI})$ is sensibly an increasing function of mass flux and a decreasing function of system pressure (Figure 4). Between the infinite possible correlations, we have chosen the following simple one, obtained by minimizing the error with respect to experimental data:

$$
x_{I}=(0.44-0.0006 p) G^{0.114}
$$

Where $\mathrm{p}$ must be expressed in bar and $\mathrm{G}$ in $\mathrm{kg} / \mathrm{m} 2 \mathrm{~s}$.

The comparison between experimental data and equation (1) predictions is reported in Figure 10.

Equation (1) reproduces the 12 data on first quality dryout with a root mean square error of $2.4 \%$.

As previously said in the introduction, first quality dryout occurrence in well-established annular flow for high qualities and low heat fluxes must be considered a global phenomenon not influenced (or only slightly) by the heat flux distribution along the channel, but only to the total power given to the flowing mixture. Liquid film rupture must be considered a phenomenon related to the thermal history of the flowing mixture, in the sense that, at fixed pressure and mass flux, only the total power given to the mixture determines the quality in correspondence of which the liquid film brakes causing the drying of the tube surface.

Experiences conduced in the past at CISE labs have shown the validity of the global approach for dryout type crisis by comparing two different power distributions applied to a channel with the same mass flux, pressure and inlet subcooling [16]. A tube of nearly 1.1 meters length was firstly powered with an initial tract of 1 meter with a low heat flux and the last portion of the tube $(0.1$ meters) with a high heat flux. The same tube, in the same thermohydraulics conditions, was tested with the reversed conditions, i.e. a first short tract $(0.1$ meters $)$ with high heat flux and a second long tract $(1 \mathrm{~m})$ with low heat flux. What experiences have shown is that, for the two configurations explored, dryout develops for the same total power given to the fluid, regardless of the power flux distribution. This results holds for maximum heat fluxes in the short tract up to $2.5 \mathrm{MW} / \mathrm{m} 2$ and for maximum ratios of the maximum heat flux to the mean heat flux of 4 , ceasing to be valid for higher heat fluxes, probably due to the local effects that surely influence the physics of dryout at high heat fluxes.

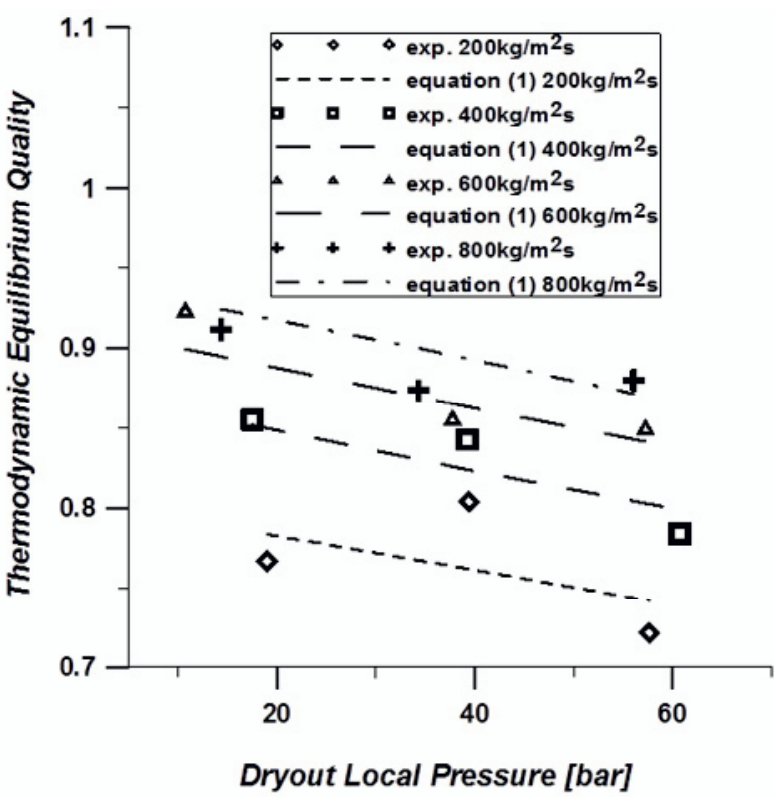

Figure 10. Comparison between equation (1) predictions and exp. results

The global hypothesis approach has a fundamental drawback on dryout prediction and modelling. In fact as far as the quality dryout can be predicted with a correlation like equation (1) as a function of pressure and mass flux, the dryout power and the dryout mean heat flux are immediately evaluated with an energy balance, regardless the fact that the heat flux distribution is uniform, as in our experiences, or a strongly varying function of tube abscissa as in a real fluid heated steam generator.

The link between first dryout quality (xI) and first dryout power (WI), i.e. the total power that must be given to the tube in order to induce dryout, is simply obtained with a power balance across the boiling channel:

$W_{I}=W_{I}^{s u b}+W_{I}^{\text {sat }}=\Gamma\left(h_{l s}\left(p_{\text {crisis }}\right)-h_{\text {in }}\right)+\Gamma h_{l v}\left(p_{\text {crisis }}\right) x_{I}$

Being $W_{I}^{\text {sub }}$ the power to be given to the subcooled fluid entering the channel in order to reach liquid saturation, $W_{I}^{\text {sat }}$ the remaining power from saturated liquid to dryout occurrence. All the thermophysical properties must be evaluated at the local pressure in the point in which thermal crisis develops (nearly the end of the heated length in our experimental facility). Introducing the inlet subcooling quality for the channel (xin) in equation (2), we obtain for the first dryout power:

$W_{I}=\Gamma h_{l v}\left(p_{\text {crisis }}\right)\left(x_{I}-x_{\text {in }}\right)$

In which $\mathrm{xI}$ must be calculated with equation (1).

Equation (3), if the heat flux is uniform, can be converted into a relation between heat flux and quality by simply dividing it by tube inner surface $(\pi d L)$. 


\subsection{Comparison with literature}

In order to compare our experimental data with literature available correlations we have chosen three correlations, namely the ones due to Ruffel [17], Ünal et alii [12] and Jayanti \& Berthoud [7].

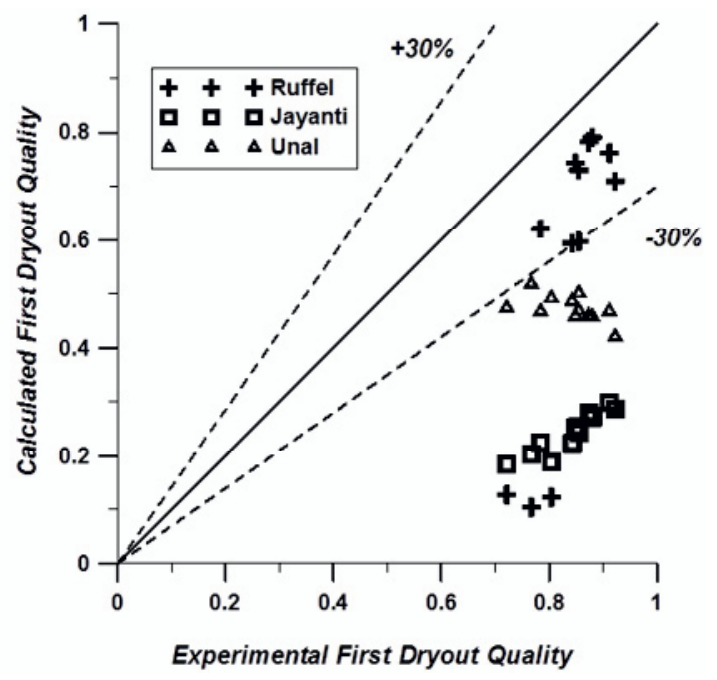

Figure 11. Comparison between experimental results and literature correlations

It is worth mentioning that, with the exception of Ruffel's correlation, all the other proposed for comparison have been obtained in a spectrum of thermohydraulics conditions significantly different than our explored conditions.

The best predictions are due to Ruffel's correlation ( $\bar{\varepsilon}=-35.1 \% ; R M S=45.6 \%$, with the exception of the three data obtained with the lower experienced mass flux $(200 \mathrm{~kg} / \mathrm{m} 2 \mathrm{~s})$ that are out of Ruffel's correlation validity boundaries.

All the other correlations significantly under predict the experimental results $\left(\bar{\varepsilon}_{\text {Unal }}=-43.3 \% ; R M S_{\text {Unal }}=43.7 \%\right.$; $\bar{\varepsilon}_{\text {Jayanti }}=-71.2 \% ; R M S_{\text {Jayanti }}=71.3 \%$ ) as Figure shows.

\subsection{Dryout location around tube circumference and film shape hypothesis.}

Dryout onset location is an interesting parameter that allows us to make some hypothesis on film shape distribution in the coiled tube. Film shape during annular flow in coiled tubes is determined by the balance between centrifugal and gravitational forces. The physics is particularly complicated by the presence of the secondary flows in the vapour core (the upper one always run counter clockwise, while the lower one clockwise if the helix axis is on the left of tube cross section) that induce tangential stresses in the liquid film, thus influencing significantly its shape. In particular, if this tangential stresses overcome the centrifugal force on the liquid film, the liquid film flow rate is directed from the tube outer side (EXTRADOSSO) to the inner side (INTRADOSSO), resulting in an inverted annular flow, i.e. a liquid film thicker on the inner side [5].

Table 2 collect the locations around tube circumference in which first dryout were experienced.

Table 2. Dryout locations (first dryout)

$\begin{array}{ccc}\text { Pressure }^{\mathrm{a}}[\mathrm{bar}] & \text { Mass flux } \mathrm{b}^{\mathrm{b}}\left[\mathrm{kg} / \mathrm{m}^{2} \mathrm{~s}\right] & \text { Dryout location } \\ 20 & 200 & \mathrm{U} / \mathrm{E} \\ 20 & 400 & \mathrm{U} / \mathrm{D} \\ 20 & 600 & \mathrm{n} . \mathrm{a} \\ 20 & 800 & \mathrm{n} . \mathrm{a} \\ 40 & 200 & \mathrm{U} / \mathrm{E} \\ 40 & 400 & \mathrm{U} \\ 40 & 600 & \mathrm{U} / \mathrm{D} \\ 40 & 800 & \mathrm{U} / \mathrm{D} \\ 60 & 200 & \mathrm{U} \\ 60 & 400 & \mathrm{U} \\ 60 & 600 & \mathrm{U} / \mathrm{D} \\ 60 & 800 & \mathrm{U} / \mathrm{D}\end{array}$

First dryout inception always occurs, at least, at UP location. These results are probably related to a stratified situation caused by the low mass fluxes and the nearly horizontal disposition of the pipe in the helix (due to the small coil pitch). For the lowest investigated pressures, i.e. 20 and 40 bar, and for the lowest mass flux, i.e. 200 $\mathrm{kg} / \mathrm{m} 2 \mathrm{~s}$, first dryout inception occurs contemporarily at UP and EXTRA position (indicated with U/E in table 2).

Such a result shows a probable mixed situation involving a competition between stratification due to gravity and film inversion. The presence of film inversion is compatible with the low pressures that, by enhancing the slip ratio between the phases could give to the vapour phase sufficient centrifugal force for overcoming liquid one.

At the highest mass fluxes dryout inception started at UP and DOWN locations, thus indicating a probable situation in which entrainment and redeposition effects overcame the other ones.

In Figure 12 is sketched a schematic representation of film shape during the three dryout configurations experienced (from left to right U, U/E, U/D). Film thicknesses are greatly exaggerated with the scope of better representing the physical situation.

\footnotetext{
${ }^{\mathrm{a}}$ These are nominal values of the pressures in correspondence of dryout location ( $\sim$ the end of the heated length); real ones could significantly differ especially at low pressures when test section pressure drops are very high.

${ }^{\mathrm{b}}$ Nominal value.
} 

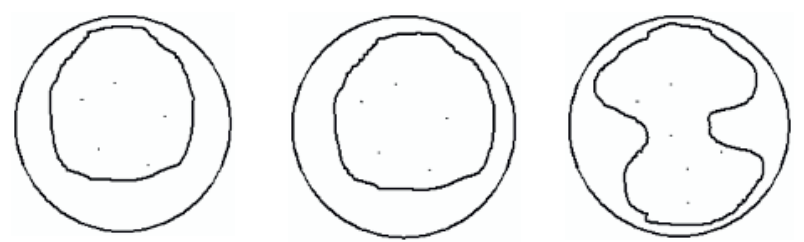

Figure 12. Argued film shape obtained from crisis results

\section{Conclusions}

Dryout occurrence has been experimentally evaluated in an helically coiled steam generator tube. The electrically heated test section represent a full scaled mean tube of the steam generator of IRIS nuclear reactor. Both first dryout and last dryout inceptions have been detected and correlated with system variables. First dryout quality resulted a monotonically increasing function of mass flux and a decreasing function of dryout local pressure. A simple correlation for predicting first dryout quality in the range of explored conditions for our test section have been proposed. Comparison of the experimental results with three correlations taken from literature showed the difficulty in predicting such an important thermohydraulic parameter in helically coiled heat exchangers.

\section{Acknowledgements}

The Authors are grateful to Gustavo Cattadori, Andrea Achilli and Stefano Gandolfi from SIET labs for their valuable collaboration and deep expertise. The Authors are grateful also to Mario Carelli and the colleagues of the IRIS project for the opportunity to work in such a stimulating and challenging adventure. This research activity has been performed in the frame of the FIRB2001 and COFIN-2004 programmes and with the financial support from the MIUR-Italian Ministry of the University and Research.

\section{Bibliography}

1. M.D. Carelli, L.E. Conway, L.Oriani, B.Petrovic, C.V.Lombardi, M.E.Ricotti, A.C.O.Barroso, Nuclear Engineering and Design 230 (2004) 151-167.

2. W.R. Dean, Note on the motion of fluid in a curved pipe, Phil. Mag. S. 7 Vol. 4. No. 20. July 1927.

3. Y.Mori, W.Nakayama, Int. J. Heat Mass Transfer 8 (1965) 67-82.

4. Y.Mori, W.Nakayama, Int. J. Heat Mass Transfer 10 (1967) 37-59.

5. G.F.Hewitt, S.Jayanti, J.Fluid Mech. 236 (1992) 497511.

6. D.W.James, Steam generator material constrains in UK design gas-cooled reactors, In Technology of steam generators for gas-cooled reactors, Proceedings of a specialists meeting organized by the International Atomic Energy Agency and held in Winterthur, Switzerland, 9-12 March 1987.

7. S.Jayanti, G.Berthoud, Nuclear engineering and design 122 (1990) 105-118.
8. CISE (Centro Informazioni Studi Esperienze), A research program in two-phase flow, January 1963. A work performed under the Euratom contract No.002-5911 RDI (CAN-1).

9. R.Roumy, Dryout in helically coiled tubes with boiling Freon-12, European Two-phase Group Meeting, Risp. (1971).

10. M.A.Styrikovich, V.S.Polonsky, V.Vreshetov, International Journal of Heat and Mass Transfer 27 (1984) 1245-1250.

11. G. Berthoud, S. Jayanti,, International journal of heat and mass transfer 33 (1990) 1451-1463.

12. H.C.Ünal, M.L.van Gasselt, P.M. van't Veerlat, International journal of heat and mass transfer 24 (1981) 285-298.

13. M.K.Jensen, A.E.Bergles, International journal of heat and mass transfer 25 (1982) 1383-1395.

14. http://www.siet.it

15. J.G. Collier, Convective boiling and condensation, McGrawHill 1981.

16. S. Bertoletti, G.P. Gaspari, C. Lombardi, G. Peterlongo, M. Silvestri, F.A. Tacconi., Energia Nucleare, 12, n.3, marzo 1965.

17. E.E. Ruffel, The application of heat transfer and pressure drop data to the design of helical coil oncethrough boilers, Symp. Multi-phase flow systems, University of Strathclyde, Inst. Chem. Eng. Symp. Ser., no38, paper 15, 1974. 